\title{
Polarization and Spectral Energy Distribution in OJ 287 during the 2016/17 Outbursts
}

\author{
Mauri Valtonen ${ }^{1,2}$, Stanislaw Zola ${ }^{3,4, *(D)}$, Helen Jermak ${ }^{5}$, Stefano Ciprini ${ }^{6,7}$, Rene Hudec ${ }^{8,9}$, \\ Lankeswar Dey ${ }^{10}$, Achamveedu Gopakumar ${ }^{10}$, Daniel L. Reichart ${ }^{11}$, Daniel B. Caton ${ }^{12}$, \\ Kosmas Gazeas ${ }^{13}$, Katsura Matsumoto ${ }^{14}$, Waldemar Ogloza ${ }^{4}$, Marek Drozdz ${ }^{4}$, \\ Fahri Alicavus ${ }^{15,16}$, Oleksandr Baransky ${ }^{17}$, Andrei Berdyugin ${ }^{2}$, Panos Boumis ${ }^{18}$, \\ Yurii Bufan 19,20, Bartlomiej Debski ${ }^{3}$, Huseyin Er ${ }^{21}$, Ahmet Erdem 15,16, Vira Godunova ${ }^{19}$, \\ Shirin Haque ${ }^{22}$, Vivian L. Hoette ${ }^{23}$, Jan Janik 24 (D), Mark Kidger ${ }^{25}$, Tomasz Kundera ${ }^{3}$,
} Sebastian Kurowski ${ }^{3}$, Alexis Liakos ${ }^{18}$ iD, Isa Mohammed ${ }^{26}$, Kari Nilsson ${ }^{1}$, Urszula Pajdosz ${ }^{3}$, Vilppu Piirola 1,2 , Tapio Pursimo ${ }^{27}$, Brandon Rajkumar ${ }^{22}$, Andrii O. Simon ${ }^{28}$, Michal Siwak ${ }^{4}$, Eda Sonbas ${ }^{29}$, Ian A. Steele ${ }^{5}$, Volodymir V. Vasylenko ${ }^{28}$, Michal Zejmo ${ }^{30}$ and Pawel Zielinski ${ }^{24}$

1 Finnish Centre for Astronomy with ESO, University of Turku, 21500 Kaarina, Finland; mvaltonen2001@yahoo.com (M.V.); kari.nilsson@utu.fi (K.N.); piirola@utu.fi (V.P.)

2 Tuorla Observatory, Department of Physics and Astronomy, University of Turku, 21500 Kaarina, Finland; andber@utu.fi

3 Astronomical Observatory, Jagiellonian Unviersity, ul. Orla 171, 30-244 Krakow, Poland; bartek@oa.uj.edu.pl (B.D.); kundera@oa.uj.edu.pl (T.K.); sebastian@oa.uj.edu.pl (S.K.); urszula.pajdosz@gmail.com (U.P.)

4 Mt. Suhora Observatory, Pedagogical University, ul. Podchorazych 2, 30-084 Krakow, Poland; sfogloza@cyf-kr.edu.pl (W.O.); sfdrozdz@cyf-kr.edu.pl (M.D.); siwak@oa.uj.edu.pl (M.S.)

5 Astrophysics Research Institute, Liverpool John Moores University, IC2, Liverpool Science Park, Brownlow Hill L3 5RF, UK; H.E.Jermak@ljmu.ac.uk (H.J.); I.A.Steele@ljmu.ac.uk (I.S.)

6 Space Science Data Center-Agenzia Spaziale Italiana, via del Politecnico, snc, I-00133 Roma, Italy; stefano.ciprini.asdc@gmail.com

7 Istituto Nazionale di Fisica Nucleare, Sezione di Perugia, I-06123 Perugia, Italy

8 Faculty of Electrical Engineering, Czech Technical University in Prague, Technicka 2, CZ-166 27 Praha 6, Czech Republic; rene.hudec@gmail.com

9 Engelhardt Astronomical observatory, Kazan Federal University, Kremlyovskaya street 18, Kazan 420008, Russia

10 Department of Astronomy and Astrophysics, Tata Institute of Fundamental Research, Mumbai 400005, India; lanky441@gmail.com (L.D.); gopu.tifr@gmail.com (A.G.)

11 Department of Physics and Astronomy, University of North Carolina at Chapel Hill, Chapel Hill, NC 27599, USA; dan.reichart@gmail.com

12 Dark Sky Observatory, Department of Physics and Astronomy, Appalachian State University, Boone, NC 28608, USA; catondb@appstate.edu

13 Section of Astrophysics, Astronomy and Mechanics, Department of Physics, National \& Kapodistrian University of Athens, Zografos, Athens GR-15784, Greece; kgaze@physics.auth.gr

14 Astronomical Institute, Osaka Kyoiku University, 4-698 Asahigaoka, Kashiwara, Osaka 582-8582, Japan; katsura@cc.osaka-kyoiku.ac.jp

15 Department of Physics, Faculty of Arts and Sciences, Canakkale Onsekiz Mart University, Canakkale TR-17100, Turkey; fahrialicavus@comu.edu.tr (F.A.); aerdem@comu.edu.tr (A.E.)

16 Astrophysics Research Center and Ulupinar Observatory, Canakkale Onsekiz Mart University, Canakkale TR-17100, Turkey

17 Observatory of Taras Shevshenko National University of Kyiv, Observatorna str. 3, 04053 Kyiv, Ukraine; abaransky@ukr.net

18 Institute for Astronomy, Astrophysics, Space Applications and Remote Sensing, National Observatory of Athens, Metaxa \& Vas. Pavlou St., Penteli, Athens GR-15236, Greece; ptb@noa.gr (P.B.); alliakos@noa.gr (A.L.)

19 ICAMER Observatory of NASU, 27, Acad. Zabolotnoho str., 03143 Kyiv, Ukraine; bufkoy@gmail.com (Y.B.); V_Godunova@bigmir.net (V.G.) 
20 Astronomical Observatory of National Ivan Franko University of Lviv, 8, Kyryla i Methodia str., 79005 Lviv, Ukraine

21 Department of Physics, Faculty of Science, Atatürk University, Erzurum 25240, Turkey; huseyin.er@ogr.atauni.edu.tr

22 Department of Physics, University of the West Indies, St Augustine, Trinidad; shirin.haque@gmail.com (S.H.); brandon.rajkumar@sta.uwi.edu (B.R.)

23 Yerkes Observatory, Department of Astronomy and Astrophysics, University of Chicago, 373 W. Geneva St., Williams Bay, WI 53191, USA; vhoette@yerkes.uchicago.edu

24 Department of Theoretical Physics and Astrophysics, Masaryk University, Kotlářská 2, 61137 Brno, Czech Republic; honza@physics.muni.cz (J.J.); pawel@physics.muni.cz (P.Z.)

25 Herschel Science Centre, ESAC, European Space Agency, 28692 Villanueva de la Cañada, 28692 Madrid, Spain; mkidger@sciops.esa.int

26 Caribbean Institute of Astronomy, c/o Department of Physics, University of the West Indies, St. Augustine, Trinidad; isa@caribbeanastronomy.com

27 Nordic Optical Telescope, Apartado 474, Santa Cruz de La Palma E-38700, Spain; tpursimo@not.iac.es

28 Astronomy and Space Physics Department, Taras Shevshenko National University of Kyiv, Volodymyrska str. 60, 01033 Kyiv, Ukraine; skazhenijandrew@gmail.com (A.O.S.); Vol_odya@ukr.net (V.V.V.)

29 Department of Physics, University of Adiyaman, Adiyaman 02040, Turkey; edasonbas@gmail.com

30 Janusz Gil Institute of Astronomy, University of Zielona Góra, Szafrana 2, PL-65-516 Zielona Góra, Poland; michalzejmo@gmail.com

* Correspondence: szola@oa.uj.edu.pl; Tel.: +48-12-6238-624

Received: 29 August 2017; Accepted: 9 November 2017; Published: 20 November 2017

Abstract: We report optical photometric and polarimetric observations of the blazar OJ 287 gathered during 2016/17. The high level of activity, noticed after the General Relativity Centenary flare, is argued to be part of the follow-up flares that exhibited high levels of polarization and originated in the primary black hole jet. We propose that the follow-up flares were induced as a result of accretion disk perturbations, travelling from the site of impact towards the primary SMBH. The timings inferred from our observations allowed us to estimate the propagation speed of these perturbations. Additionally, we make predictions for the future brightness of OJ 287.

Keywords: galaxies: active; BL lacertae objects: individual (OJ 287); super massive black holes

\section{Introduction}

The blazar OJ 287 is the best candidate for hosting an inspiraling SMBH binary. It exhibits high levels of optical activity roughly every 12 years, which has been observed since 1888 by using old photographic plates, and more recently, via optical photometry (Sillanpää et al. 1988 [1]; Hudec et al. 2013 [2]). One of the key features of this activity is that it comes in pairs of outbursts which are separated by at least 1.1 years, with the separation being a function of the cycle number. The flares occur at a constant phase angle, and at the opposite phase angle of a quasi-Keplerian orbit of a given precession rate for the major axis. Therefore, they occur according to a purely mathematical rule, computed from a quasi-Keplerian model of a binary black hole system with an accretion disk. This systematic behavior leads to a model of a high mass ratio $(>100)$ binary black hole system as a central engine for OJ 287 where the high brightness episodes arise from the impact of the secondary on the accretion disk of the primary (Lehto \& Valtonen 1996 [3]; Valtonen 2007 [4]; Valtonen et al. 2010 [5]). A model of the system is presented in Figure 1. 


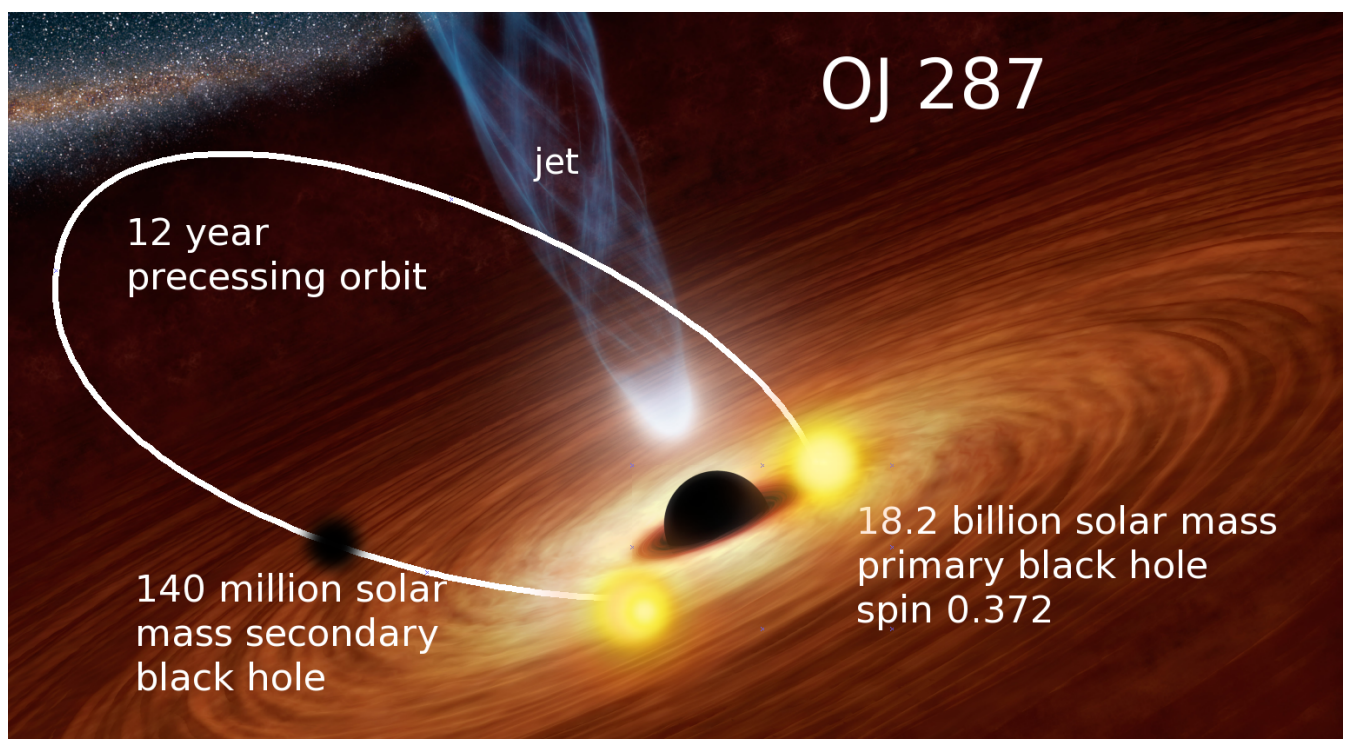

Figure 1. The improved inspiraling binary black hole central engine model for OJ 287. The current period of 12.055 year decreases by 38 days per century (Dey et al. 2017 [6]).

According to this model, the latest disk impact occurred on 2013.45. It released a hot bubble of gas, which subsequently expanded and radiated strong optical/UV bremsstrahlung radiation when it became transparent 2.42 years later (Lehto \& Valtonen 1996 [3]; Pihajoki (2016) [7]). The model predicted the beginning of the flare on $2015.96 \pm 0.12$, the exact value depending on the normalized spin value of the primary. A high spin, $\chi=0.36$, predicted an early flare while a low spin, $\chi=0.20$, predicted a later flare (Valtonen et al. 2010 [5]). The flare began rather early, on 25 November 2015, on the exact centenary of the General Relativity theory (hence its name). With this flare timing, together with improvements in the gravitational wave modeling of OJ 287, a new orbit has been calculated which gives the spin value of $\chi=0.372$ (Dey et al. 2017 [6]). The Centenary Flare was essentially unpolarised and increased the flux only in the optical/UV region. This is in contrast to typical flares in OJ 287, where a power-law spectrum rises in unison all the way from the optical to $\mathrm{X}$-rays, and generally speaking the degree of optical polarization rises together with the flux. Since the General Relativity Centenary flare, OJ 287 has stayed at 30 year record levels in optical brightness, divided into two episodes in 2016. Here we report optical photometry and polarimetry showing its 2016-2017 behavior. The follow-up flares in 2016 had high levels of polarization, and the outburst energy extended to X-rays with an almost constant optical/X-ray spectral index of $\alpha_{v}=2.7 \pm 0.1$, where flux density $f_{v} \propto v^{-\alpha_{v}}$.

While the Centenary flare arises directly as the result of the impact of the secondary black hole onto the accretion disk of the primary, the follow-up flares originate in the jet of the primary. The secondary $\mathrm{BH}$ affects the disk indirectly via tidal forces causing some of the matter in the disk to escape vertically.

The response of the accretion disk to the orbiting secondary $\mathrm{BH}$ has been calculated by Sundelius et al. (1997) [8] using non-interacting test particles to model the disk, and by Pihajoki et al. (2013) [9] using particles interacting hydrodynamically. In this paper we refer to the latter simulation, which is summarized in Figure 4 of Pihajoki et al. (2013) [9]. Separate panels show the number of particles accreted by the primary $\mathrm{BH}$ as a function of time, the number of particles accreted by the secondary, and the number of particles which escape vertically from the disk. By this calculation, the escape of particles vertically from the disk starts on 2014.2, reaches the maximum rate on 2014.6, then falls to the first minimum on 2015.1, the second peak occurs on 2015.3, after which the escape rate declines until it is close to zero in 2018.

The rise in brighness must occur after the vertical escape time since the density perturbations have to travel to the jet. Our recent experiments with non-interacting test particles show that the first 
peak comes from the inner regions of the disk, closer than the impact point to the center of the disk, while the second peak has a strong contribution from the disk beyond the impact point. This physical separation of the source regions, and their phase difference, is the reason for the double peak structure of the particles escape rate. The escaped matter may contribute to the synchrotron component of the radiation if it is accreted towards the primary and ends up in the jet. So far the simulations cannot model the subsequent fate of the vertically escaped particles; here we consider the observational evidence in this regard.

The Centenary Flare and the subsequent synchrotron flares arise by two separate processes. There is no simple rule that associates the two types of flare, nor a standard outburst light curve that includes both types of flare. It is necessary to calculate the full light curve for each flare episode individually, as done by Pihajoki et al. (2013) [9].

The new observations allow the determination of the propagation speed. We also present the prediction of the blazar brightness in the coming years.

\section{Observations}

\subsection{Photometry}

Predicted by the SMBHs binary model, the Centenary flare in OJ 287 was well covered in the optical band by a campaign with more than 20 participating sites (Valtonen et al. 2016 [10]). After this event we continued the monitoring of the blazar at several sites including the SKYNET Robotic Telescope Network achieving almost daily coverage. Observations were stopped for the summer 2016 when OJ 287 was in conjuction with the Sun and we resumed monitoring its brightness as soon as it became accessible at the end of August 2016, taking data in the wide band R filter. The observations were reduced soon after they were taken by calibrating the frames for bias, dark and flatfield. Data taken with the SKYNET telescopes were calibrated with the network pipeline. Magnitudes were extracted using the aperture method with the CMunipack program (Hroch 1998 [11]) which is a graphical interface for the DAOPHOT II code. The photometric light curve of OJ 287 taken using the wide band $R$ filter is presented in Figure 2 .

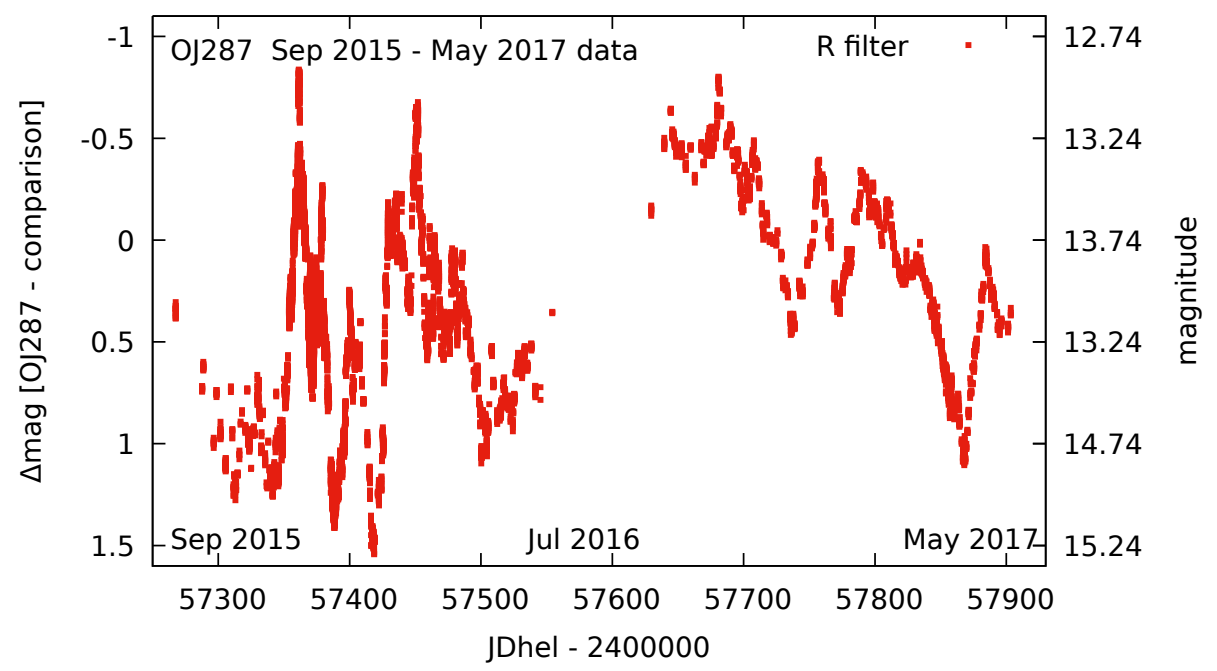

Figure 2. Light curve of OJ 287 observed during the 2015/16 and 2016/17 seasons. The gap in summer 2016 is due to OJ 287's conjunction with the Sun. 


\subsection{Polarimetry}

The fully autonomous and robotic $2 \mathrm{~m}$ Liverpool Telescope (LT) simultaneously houses 7 instruments, and any one can be accessed on a given night (Steele et al. (2004) [12]). RINGO3 is the current polarimeter on the LT (Arnold et al. (2012) [13]). It consists of a rotating Polaroid (at $0.4 \mathrm{~Hz}$ ) which modulates the incoming beam of light. The light is then split into three wavebands using dichroic mirrors, $350-640 \mathrm{~nm}$, 650-750 $\mathrm{nm}$ and 760-1000 nm. Images are captured at 8 different Polaroid rotor angles by three electron multiplying charge coupled device (EMCCD) cameras (one for each waveband). The measured counts at the 8 different Polaroid angles are combined using equations from Clarke \& Neumayer (2002) [14] to calculate the Linear Stokes Parameters $\mathrm{Q}$ and $\mathrm{U}$, whilst stacking the 8 images from the different rotor angles gives the Stokes Parameter I. Using the Linear Stokes Parameters the degree of polarization and polarization angle can be calculated as described in Clarke \& Neumayer (2002) [14] with details of the reduction process for Ringo3 data given in Jermak et al. (2016) [15]. The measurements performed for OJ 287 (linear polarization degree and polarization angle) are shown in Figure 3. In this figure we also plotted data reported in Valtonen et al. 2016 [10] and new measurements taken in 2016/17 season in the $\mathrm{R}$ filter with the Dipol-2 polarimeter on the T60 telescope in Maui, Hawaii. Detailed description of the observation routine and data reduction procedure for Dipol-2 can be found in Kosenkov et al. 2017 [16]. Except for the December 2015 flare (marked by the red vertical line), for which the polarization degree was at the level of 0.1 or below, the subsequent brightenings were highly polarized. It is interesting to note that the sudden drop of polarization has occurred on 24 January 2017 (JD 2457778). At the same time the brightness reached a local minimum and started to increase up again (see Figure 2).

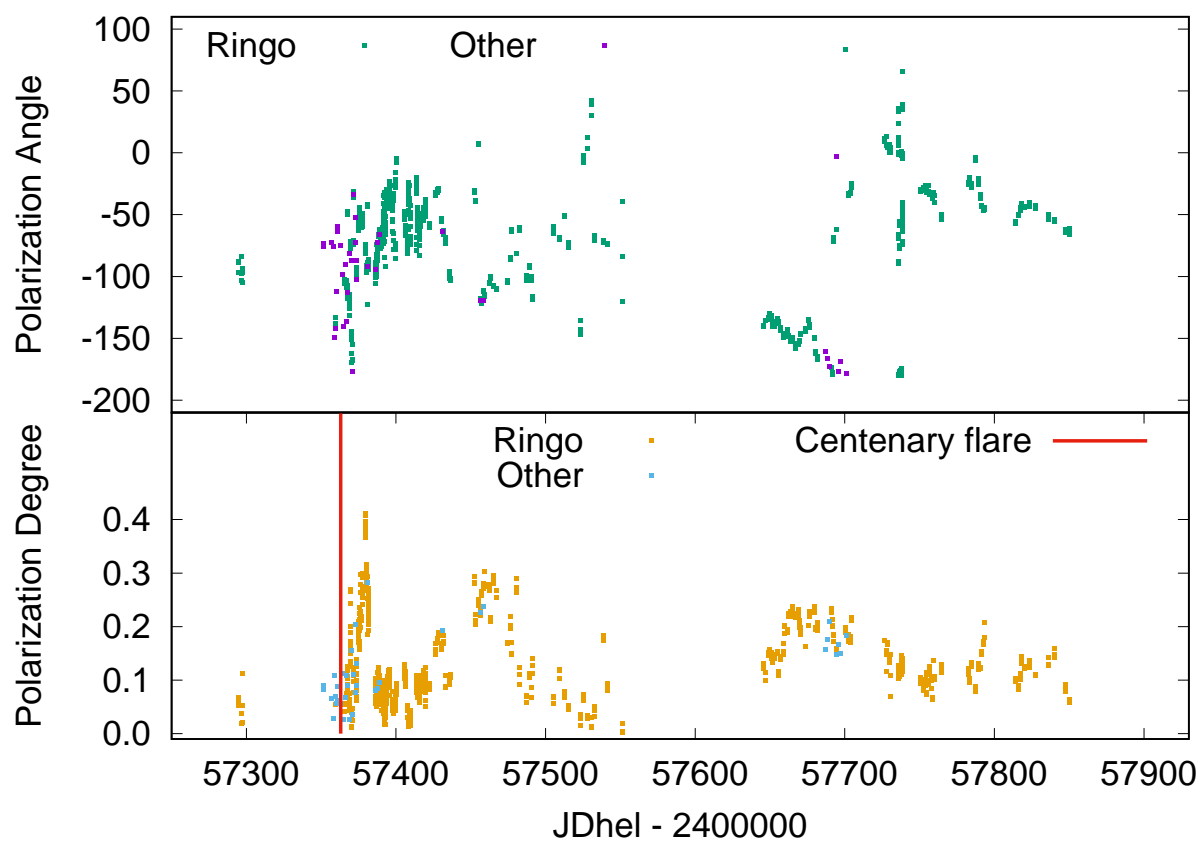

Figure 3. Polarimetric data for OJ 287 from September 2015 to May 2017.

\section{Propagation of Accretion Disk Perturbations}

We started the 2015/2016 observing campaign for the purpose of getting accurate data on the outburst predicted to occur during December 2015. The optical flux rose above its normal level of variations around 14.5 mag in $R$ band, on the exact date of the Centenary of General Relativity; thus its name Centenary flare (Valtonen et al. 2016 [10]). For the first time, this campaign obtained polarization and UV/X-ray data over the whole light curve of an OJ 287 primary impact flare, in addition to optical photometry. The campaign has continued in order to monitor the follow-up flares which have been observed as a rule in OJ 287 previous outburst episodes (Sillanpää et al. 1996a [17]; Sillanpää et al. 
1996b [18]; Valtonen et al. 2009 [19]; Valtonen et al. (2012) [20]; Pihajoki et al. (2013) [9]). While the first flare, which comes directly from the impact, is of low polarization and is limited to the optical/UV region (Valtonen et al. 2008 [21]; Valtonen \& Ciprini 2012 [22]), the follow-up flares are highly polarized and extend to the X-ray region of the spectrum (Smith et al. (1985) [23]; Seta et al. (2009) [24]). Therefore in the current campaign both polarization measurements and UV/X-ray data have had a central role in determining the likely origin of the different light curve features.

Prior to this campaign the speed of the transmission of perturbations from the impact site to the jet was an open question. In the 1994/1996 flares it was estimated that it takes 0.25 years for the transit (Valtonen et al. 2006 [25]), while for the 2007 flare the transit time was 0.02 years (Valtonen et al. 2009 [19]). From these two data points alone it is difficult to determine the transmission speed, even though qualitatively they are consistent with the greater distance of the 1994/1995 impacts (about $5500 \mathrm{AU}$ on average) from the center of the accretion disk than the distance of the $2007 \mathrm{impact}$ point (about $3200 \mathrm{AU}$ from the center). One solution based on these data is that the transit speed is about $(1+z) / 6$ in units of $\mathrm{c}$ down to $3000 \mathrm{AU}$ ( $\mathrm{z}$ being the object's redshift of 0.306 ), at which point the perturbation is quickly transmitted to the jet. The transit time is then $=$ (distance from the center in $1000 \mathrm{AU}-3) / 10$ years. Using this formula, we expect the transit time from the 2013 impact location (at the distance of about 18,000 AU from the center) to be 1.5 years, and the two main peaks in the follow-up light curve occur at 2016.1 (JD 2457424) and 2016.8 (JD 2457679), both with a rather broad ( \pm 0.2 year) width (Pihajoki (2016) [7]). Thus the follow-up outbursts were expected very soon after the primary outburst at 2015.87, and they were anticipated to carry on through April 2016, and then reappear around August 2016 and last until the end of 2016.

Using the Pihajoki et al. (2013) [9] model, we can compare the blazar observed brightness level after the December 2015 flare, in 2016 and 2017, as the result of disturbances propagating through the disk towards the primary SMBH. This comparison as well as the predicted behaviour in the future is shown in Figure 4. We would like to point out that the strength of the second jet outburst at the end of 2016 (JD $\approx 2,457,690$ ) is underestimated in the model of Pihajoki et al. (2013) [9]. This is due to an artificial truncation of the accretion disk at 21,600 AU in the model. Without this truncation, the late 2016 outburst theoretically would have a somewhat greater brightness than the early 2016 (JD $\approx 2,457,450$ ) one.

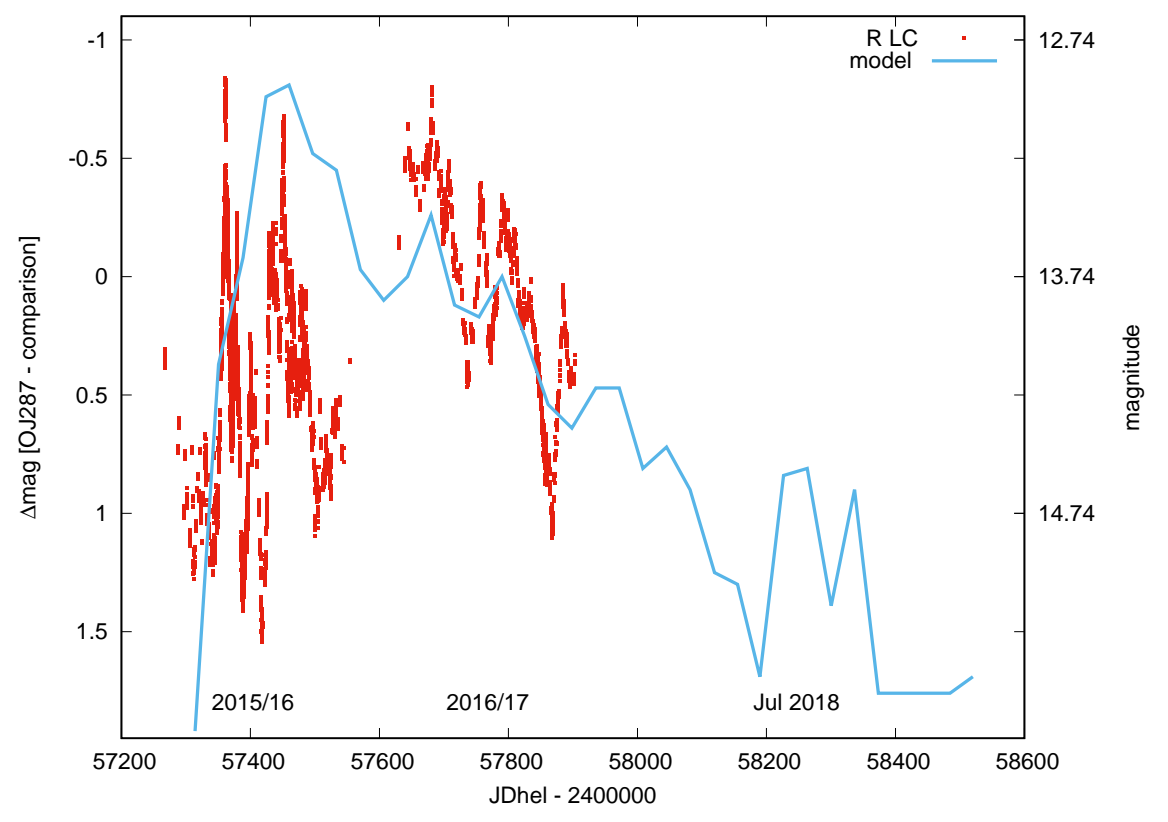

Figure 4. Prediction of OJ 287 brightness changes. 


\section{Conclusions}

We have observed the expected two peaks in the follow-up light curve of OJ 287. They are both highly polarized in optical and both the optical and X-ray spectrum are consistent with the synchrotron radiation. It is therefore likely that the emission originates in the jet of the primary black hole. The transmission time of the perturbations is confirmed as 1.5 years. Since the distance for the perturbation to travel is $15,000 \mathrm{AU}$, the speed is $\sim(1+z) / 6$ in units of $\mathrm{c}$. It has been previously estimated that this is the sound speed in the corona of the accretion disk (Pihajoki et al. (2013) [9]); thus it appears that the perturbations are transmitted via the corona to the inner parts of the accretion disk at about $3000 \mathrm{AU}(\sim 8.3$ Schwarzschild radii of the primary $\mathrm{BH})$. From there, the perturbations appear to influence the jet without further delay.

Acknowledgments: This work was partially supported by the NCN grant No. 2013/09/B/ST9/00599. R.H. acknowledges GA CR grant 13-33324S. F.A. and A.E. thank the Scientific and Technological Research Council of Turkey (TUBITAK) for a partial support in using T60 telescope with project numbers 10CT60-76 and 16DT60-1115. Authors are grateful to the Institute for Astronomy, University of Hawaii (IfA), for the allocation of IfA time for DIPOL-2 observations at the UH88 and T60 telescopes.

Author Contributions: M.V., A.G. and L.D. did GR computations, S.Z. coordinated the optical observing campaign and reduced the data, S.C. gathered and reduced the UV and X-ray observations, H.J. gathered polarimetric data with the LT, everyone else contributed photometric data.

Conflicts of Interest: The authors declare no conflict of interest.

\section{Abbreviations}

The following abbreviations are used in this manuscript:

$\mathrm{BH} \quad$ black hole

$\mathrm{SMBH}$ super massive black hole

JDhel Heliocentric Julian Day

PA polarization angle

PD polarization degree

\section{References}

1. Sillanpää, A.; Haarala, S.; Valtonen, M.J.; Sundelius, B.; Byrd, G.G. OJ 287-Binary pair of supermassive black holes. Astrophys. J. 1988, 325, 628-634.

2. Hudec, R.; Bašta, M.; Pihajoki, P.; Valtonen, M. The historical 1900 and 1913 outbursts of the binary blazar candidate OJ 287. Astron. Astrophys. 2013, 559, A20.

3. Lehto, H.J.; Valtonen, M.J. OJ 287 Outburst Structure and a Binary Black Hole Model. Astrophys. J. 1996, 460, 207-213.

4. Valtonen, M. New Orbit Solutions for the Precessing Binary Black Hole Model of OJ 287. Astrophys. J. 2007, $659,1074-1081$.

5. Valtonen, M.J.; Mikkola, S.; Merritt, D.; Gopakumar, A.; Lehto, H.J.; Hyvönen, T.; Rampadarath, H.; Saunders, R.; Basta, M.; Hudec, R. Measuring the Spin of the Primary Black Hole in OJ 287. Astrophys. J. 2010, 709, 725-732.

6. Dey, L.; Valtonen, M.J.; Zola, S.; Ciprini, S.; Gopakumar, A.; Matsumoto, K.; Sadakane, K.; Kidger, M.; Gazeas, K.; Nilsson, K.; et al. GR Centenary flare in OJ287: Improved orbital parameters. In preparation.

7. Pihajoki, P. Black hole accretion disc impacts. Mon. Not. R. Astron. Soc. 2016, 457, 1145-1161.

8. Sundelius, B.; Wahde, M.; Lehto, H.J.; Valtonen, M.J. A Numerical Simulation of the Brightness Variations of OJ 287. Astrophys. J. 1997, 484, 180-185.

9. Pihajoki, P.; Valtonen, M.; Zola, S.; Liakos, A.; Drozdz, M.; Winiarski, M.; Ogloza, W.; Koziel-Wierzbowska, D.; Provencal, J.; Nilsson, K.; et al. Precursor Flares in OJ 287. Astrophys. J. 2013, 764, 5-14.

10. Valtonen, M.J.; Zola, S.; Ciprini, S.; Gopakumar, A.; Matsumoto, K.; Sadakane, K.; Kidger, M.; Gazeas, K.; Nilsson, K.; Berdyugin, A.; et al. Primary Black Hole Spin in OJ 287 as Determined by the General Relativity Centenary Flare. Astrophys. J. 2016, 819, L37-L43. 
11. Hroch, F. Computer Programs for CCD Photometry. In Proceedings of the 29th Conference on Variable Star Research, Brno, Czech Republic, 7-9 November 1997; p. 30.

12. Steele, I.A.; Smith, R.J.; Rees, P.C.; Baker, I.P.; Bates, S.D.; Bode, M.F.; Bowman, M.K.; Carter, D.; Etherton, J.; Ford, M.J.; et al. The Liverpool Telescope: Performance and first results. Proc. SPIE 2004, 5489, 679-692.

13. Arnold, D.S.; Steele, I.A.; Bates, S.D.; Mottram, C.J.; Smith, R.J. RINGO3: A multi-colour fast response polarimeter. Proc. of SPIE 2012, doi: 10.1117/12.927000.

14. Clarke, D., Neumayer, D. Experiments with a novel CCD stellar polarimeter. Astron. Astrophys. 2002, 383, 360-366.

15. Jermak, H.; Steele, I.A.; Lindfors, E.; Hovatta, T.; Nilsson, K.; Lamb, G.P.; Mundell, C.; de Almeida, U.B.; Berdyugin, A.; Kadenius, V.; et al. The RINGO2 and DIPOL optical polarization catalogue of blazars. Mon. Not. R. Astron. Soc. 2016, 462, 4267-4299.

16. Kosenkov, I.A.; Berdyugin, A.V.; Piirola, V.; Tsygankov, S.S.; Pallé, E.; Miles-Páez, P.A.; Poutanen, J. High-precision optical polarimetry of the accreting black hole V404 Cyg during the 2015 June outburst. Mon. Not. R. Astron. Soc. 2017, 468, 4362-4373.

17. Sillanpää, A.; Takalo, L.O.; Pursimo, T.; Lehto, H.J.; Nilsson, K.; Teerikorpi, P.; Heinaemaeki, P.; Kidger, M.; de Diego, J.A.; Gonzalez-Perez, J.N.; et al. Confirmation of the 12-year optical outburst cycle in blazar OJ 287. Astron. Astrophys. 1996, 305, L17-L20.

18. Sillanpää, A.; Takalo, L.O.; Pursimo, T.; Lehto, H.J.; Nilsson, K.; Teerikorpi, P.; Heinaemaeki, P.; Kidger, M.; de Diego, J.A.; Gonzalez-Perez, J.N.; et al. Double-peak structure in the cyclic optical outbursts of blazar OJ 287. Astron. Astrophys. 1996, 315, L13-L16.

19. Valtonen, M.; Nilsson, K.; Villforth, C.; Lehto, H.J.; Takalo, L.O.; Lindfors, E.; Sillanpää, A.; Hentunen, V.; Mikkola, S.; Zola, S.; et al. Tidally Induced Outbursts in OJ 287 during 2005-2008. Astrophys. J. 2009, 698, 781-785.

20. Valtonen, M.; Ciprini, S.; Lehto, H.J. On the masses of OJ 287 black holes. Mon. Not. R. Astron. Soc. 2012, 427, 77-83.

21. Valtonen, M.; Lehto, H.J.; Nilsson, K.; Heidt, J.; Takalo, L.O.; Sillanpää, A.; Villforth, C.; Kidger, M.; Poyner, G.; Pursimo, T.; et al. Massive binary black-hole system in OJ 287 and a test of general relativity. Nature 2008, 452, 851-853.

22. Valtonen, M.; Ciprini, S. OJ 287 binary black hole system. Mem. S.A.It. 2012, 75, 282-287.

23. Smith, P.S.; Balonek, T.J.; Heckert, P.A.; Elston, R.; Schmidt, G.D. UBVRI field comparison stars for selected active quasars and BL Lacertae objects. Astron. J. 1985, 90, 1184-1187.

24. Seta, H. Suzaku and Multi-Wavelength Observations of OJ 287 during the Periodic Optical Outburst in 2007. Publ. Astron. Soc. Jpn. 2009, 61, 1011-1022.

25. Valtonen, M.; Lehto, H.J.; Sillanpää, A.; Nilsson, K.; Mikkola, S.; Hudec, R.; Basta, M.; Teräsranta, H.; Haque, S.; Rampadarath, H. Predicting the Next Outbursts of OJ 287 in 2006-2010. Astrophys. J. 2006, 646, 36-48.

(C) 2017 by the authors. Licensee MDPI, Basel, Switzerland. This article is an open access article distributed under the terms and conditions of the Creative Commons Attribution (CC BY) license (http:/ / creativecommons.org/licenses/by/4.0/). 